\title{
Acute Changes in Postural Control after Soccer Heading
}

Authors

Affiliations
F. J. Haran ${ }^{1}$, R. Tierney ${ }^{1}$, W. G. Wright ${ }^{2}$, E. Keshner ${ }^{2}$, M. Silter ${ }^{1}$

${ }^{1}$ Kinesiology, Temple University, Philadelphia, United States

${ }^{2}$ Physical Therapy, Temple University, Philadelphia, United States
Key words

concussion

- MTBI

- balance

- stability

virtual environment

accepted after revision January 27, 2012

Bibliography DOI http://dx.doi.org/ 10.1055/s-0032-1304647 Published online: November 22, 2012 Int J Sports Med 2013; 34 : 350-354 @ Georg Thieme Verlag KG Stuttgart · New York ISSN 0172-4622

Correspondence

Dr. F. J. Haran, PhD Kinesiology

Temple University

120 Pearson Hall

19122 Philadelphia

United States

Tel.: +1/215/204 4001

Fax: $+1 / 215 / 2044414$

fjharan@gmail.com

\section{Abstract}

$\nabla$

This study intended to determine if an acute bout of soccer heading alters postural control and pronounced self-reported symptoms of cerebral concussion. Collegiate soccer players were randomly assigned to one of 2 groups. Each participant completed a baseline postural control assessment prior to heading. Participants either simulated (control group; CG) or performed (experimental group; EG) 10 headers at $11.2 \mathrm{~m} / \mathrm{s}$ in $10 \mathrm{~min}$. The postural assessment was repeated post heading at hrs 1,24 , and 48 . The postural

\section{Introduction}

Soccer is unique in sport as the unprotected forehead is actively impacted to stop and redirect the ball. Known as a "header", the impact on the head is believed to expose the brain to subconcussive trauma due to rapid deceleration of the skull post impact [7]. Whether heading results in neurological dysfunction similar to a concussion has yet to be determined.

Research has shown a significant increase of biochemical markers of neurological damage following a soccer match which correlates with the number of headers taken $[25,26]$. Other research has focused on the build-up of neuropsychological effects after long-term play, however, several research groups have reported chronic cognitive deficits in experienced players $[6,15,-20,31-33]$ while others have reported the opposite $[1,16,18,27,28]$. The discrepancy may be attributed to methodological differences: lack of baseline data, failure to control confounding variables (e.g., alcohol abuse), selection bias, over reliance on self-report, and type I error inflation.

Research into short-term effects of a single bout of heading has identified no acute deficits in cognition [22] or balance $[4,17,20]$; however, there are a number of reasons why they may have been control parameter assessed was the root mean square (RMS) of the center of mass (COM). COM RMS were calculated for the anterior-posterior (AP) and medial-lateral (ML) time series. Compared to the CG, for the AP and ML time series COM RMS values were significantly higher in the EG at hr 24 ( p < 0.05). An acute bout of heading results in quantifiable alterations in postural control that are detectable $24 \mathrm{~h}$ post heading and dissipate within an additional $24 \mathrm{~h}$. The significant findings may be due to the dynamic postural control assessment that incorporated robust discordant environmental conditions. missed. Some studies did not look for such deficits, while others failed to control for ball velocity, verify sensitivity of the postural assessment, or test for statistical validity threats.

The present study was designed to examine the effect of soccer heading on postural stability by testing postural control under static and dynamic environmental conditions. To do this, athletes before and after a bout of actual or simulated headers were exposed to dynamic virtual environment [VE] combined with an unstable surface. The goal of the study was to investigate the impact of heading on neurological function using strict control of heading bouts, sensitive outcome measures, and conditions designed to increase specificity, with the aim of providing recommendations that will increase player safety. We hypothesized that athletes in the acute stage of injury would exhibit instability vs. controls when in the unpredictable, dynamic environment.

\section{Methods $\nabla$}

\section{Subjects}

16 volunteers, randomly assigned to an experimental group (EG, $\mathrm{n}=8,21 \pm 2$ years, $173 \pm 9 \mathrm{~cm}$, $70 \pm 10 \mathrm{~kg}, 6$ males, 2 females) or control group 
(CG, $\mathrm{n}=8,23 \pm 3$ years, $180 \pm 9 \mathrm{~cm}, 76 \pm 13 \mathrm{~kg}, 6$ males, 2 females), were included if they were a club or collegiate soccer team member with at least 5 years of heading experience and excluded if there was any history of head, neck, or face injury in the previous 6 months. Subjects were told to refrain from substances that could affect their nervous systems (e.g., stimulants and/or depressants) during the period of testing. All subjects signed university IRB approved informed consent and HIPAA forms [12].

\section{Soccer heading model}

An established standardized head impact model was used, consisting of a machine (JUGS Sports International, Tualatin, OR) capable of projecting a soccer ball as if kicked [13]. Balls were projected at $40^{\circ}$ from horizontal at $11.2 \mathrm{~m} / \mathrm{s}$ ( $25 \mathrm{mph}$ ). Subjects stood $10 \mathrm{~m}$ from the machine and were instructed to head the ball back in the air towards the machine.

\section{Postural assessment}

3D kinematic data were captured via a 6-camera infrared motion analysis system (Motion Analysis Corp., Santa Rosa, CA) at $120 \mathrm{~Hz}$ and low-pass filtered at $4 \mathrm{~Hz}$ using a $4^{\text {th }}$ order Butterworth filter. Reflective markers were placed on the body to derive whole body center of mass (COM) motion ( $\bullet$ Fig. 1 ), by applying a theoretical model [11] in custom written code (Mathworks, Natick, MA).

During quiet stance, subjects stood on a dynamic 3 degree-offreedom posture platform (Neurocom International Inc., Clackamas OR) and were exposed to different conditions. The platform was situated within a 3-wall VE back-projection system [24]. The assessment consisted of 6 environmental conditions: (1) stationary VE with a stationary support surface, (2) in the dark with a moving support surface, (3) rotating VE with a stationary support surface, (4) rotating VE with a moving support surface, (5) in the dark with a stationary support surface, and (6) stationary VE with a moving support surface. In conditions 4 and 6, COM motion was used to simultaneously drive platform translation in the anteroposterior (AP) and mediolateral (ML) directions, and tilt about the ML axis. Gain was adjusted to the subject's comfort level. A gain of 1.0 would move the platform to a maximum of $100 \%$ of COM movement in the same direction as COM. All conditions were repeated 3 times for a total of eighteen 30 s trials. The order of conditions was randomized and counterbalanced across subjects. The testing environment was controlled for noise and distraction.

\section{Experimental protocol}

4 data sessions were collected: hr 0, hr 1, hr 24, and hr 48. At hr 0, prior to heading, platform gain was set, and a baseline assessment was performed. No practice trials were allowed. After hr 0 session, subjects performed 10 headers (EG=actual headers, $\mathrm{CG}=\mathrm{a}$ heading maneuver with no ball contact) within a $10 \mathrm{~min}$ period. Postural assessments of all subjects were then repeated at hr 1 , hr 24 , and hr 48 starting roughly $60 \mathrm{~min}$ after the $10^{\text {th }}$ header.

\section{Data analysis}

Root mean square (RMS) was calculated for AP and ML components of each COM time series. RMS represents the average spread of a time series distribution relative to its mean. Higher RMS values are traditionally interpreted as greater postural instability or error [11]. 2 separate mixed-model 2 (group) $\times 4$ (time) $\times 6$ (condition) ANOVAs for AP and ML RMS with repeated measures of time and condition were performed (SPSS, Chicago, IL). Planned contrasts were used to test the a priori hypothesis. Significant interaction effects were explored using post-hoc comparisons (one-way ANOVA's and t-tests using a Bonferroni correction to adjust level of significance). Significance was set at $\alpha<0.05$.

\section{Results}

$\nabla$

\section{COM}

ANOVA revealed a group by session interaction $\left(F_{3,42}=2.907\right.$, $\mathrm{p}=0.046)$. Post-hoc comparisons revealed an increase in the EG at hr 24 compared to baseline ( $\mathrm{hr} 0, \mathrm{p}=0.018$, $\odot$ Fig. 2 ).

A condition by time interaction was found $\left(F_{15,210}=2.73\right.$, $\mathrm{p}=0.001)$, which post-hoc tests revealed was due to condition 4 only $(\mathrm{p}=0.031)$. Hrs 1,24 , and 48 were not significantly different than baseline; however, hrs 1 and 24 were higher than hr 48 ( $\mathrm{p}=0.001, \mathrm{p}=0.005$, respectively).

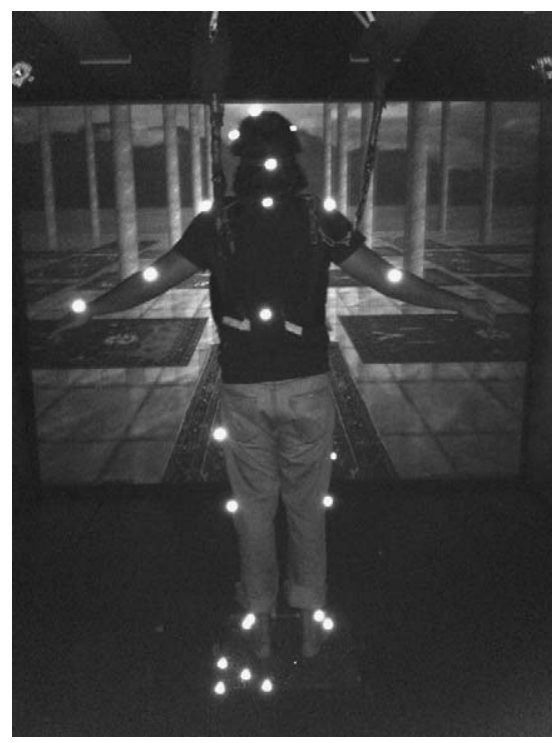

Fig. 1 Marker placement and experimental set-up.

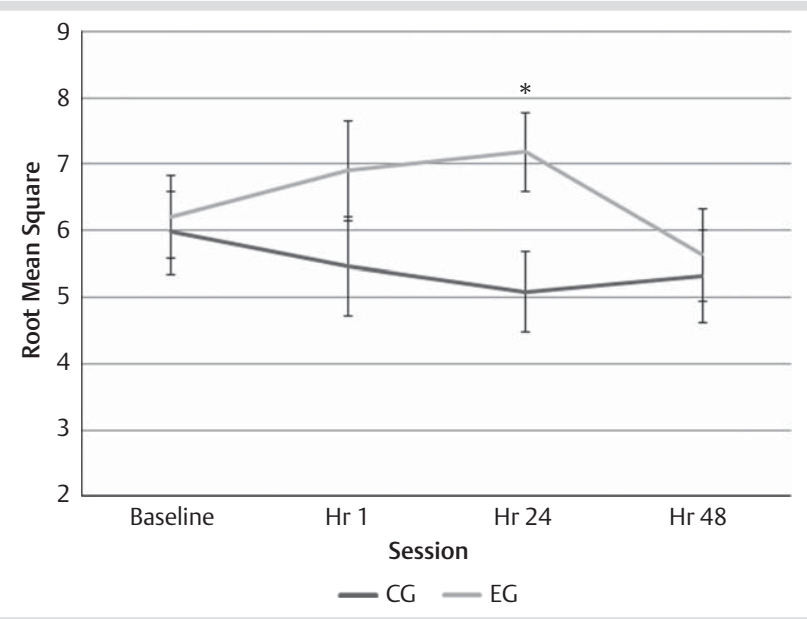

Fig. 2 ML COM group means collapsed across condition for each time period. Control $(\mathrm{N}=8)$ and experimental $(\mathrm{N}=8) . \mathrm{CG}=$ control group and $\mathrm{EG}=$ experimental group. ${ }^{*}$ Significant differences from baseline for $\mathrm{EG}$ only $(p<0.05)$. 
A significant main effect of condition was found $\left(F_{2.84,39.76}=28.14\right.$, $\mathrm{p}<0.000, \varepsilon=0.568)$. Post-hoc tests indicated conditions 2 $(\mathrm{p}=0.001), 4(\mathrm{p}<0.000)$, and $6(\mathrm{p}=0.003)$ were higher than baseline (i.e., condition 1,0 Fig. 3 ).

Planned comparisons between the most challenging condition (i.e., 4) and baseline revealed a group by session interaction, $\left(F_{3,42}=3.78, \mathrm{p}=0.017\right)$. The RMS increase from baseline to $\mathrm{hr} 24$ in the EG was significantly different from the CG $(p=0.023$, - Fig. 4).

\section{AP COM}

The main effect of time $\left(F_{3,42}=7.07, \mathrm{p}=0.001\right)$ was due to a significant RMS decrease at $\mathrm{hr} 48$ relative to the baseline $(\mathrm{p}=0.001, \diamond$ Fig. 5).

A main effect of condition $\left(F_{3,42}=13.25, \mathrm{p}=0.000, \varepsilon=0.339\right)$ was due to an increase in condition 4 relative to baseline $(\mathrm{p}=0.001, \odot$ Fig. 6$)$.

Planned comparisons between condition 4 and baseline, revealed a group by session interaction $\left(F_{3,42}=4.695, \mathrm{p}=0.006\right)$. RMS increased from baseline (hr 0 ) to hrs 1 and 24 significantly more in the EG than in the CG $(\mathrm{p}=0.033$ and $\mathrm{p}=0.040$, respectively, $\bullet$ Fig. 7).

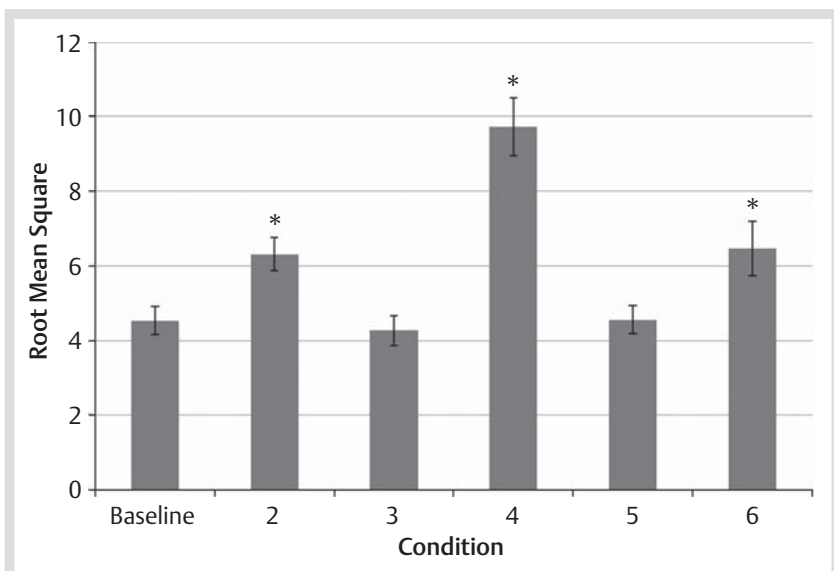

Fig. 3 ML COM means for condition collapsed across group and time. ${ }^{*}$ Significant differences from baseline $(p<0.05)$.

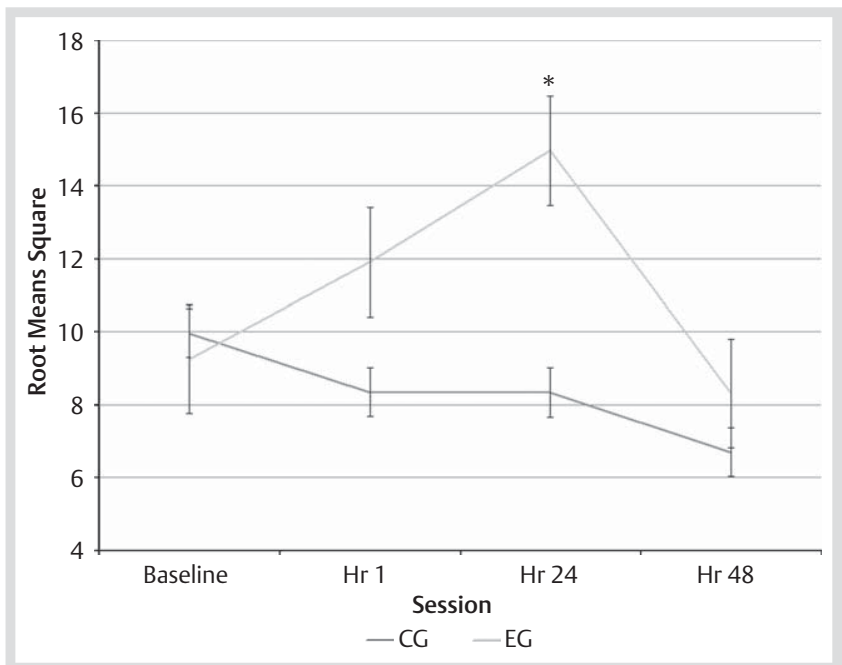

Fig. 4 ML COM group means for condition 4. * Significant differences between groups $(p<0.05)$.

\section{Discussion}

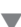

Significant findings in this study reveal that a bout of heading results in acute changes in postural control. The repetitive, subconcussive forces on the brain when heading, even at the low velocities used in this experiment, appear to cause a large enough trauma to reduce postural stability. The postural deficits found in this study appear within $1 \mathrm{~h}$ of heading and dissipate within 2 days ( $48 \mathrm{~h}$ ) with postural stability improving from baseline due to a possible practice effect that was blunted from the subconcussive head trauma. Contrary to the heading literature, the observed deficits follow a similar, yet accelerated, timeline relative to previously reported deficits associated with concussion which suggests the possibility of a subconcussive injury $[9,10]$.

Post-concussive postural instability typically appears within $24 \mathrm{~h}$ of injury and dissipates within 3-5 days [10]. It has been suggested that such deficits are a result of a sensory integration problem which prevents successful processing of visual, vestibular, and somatosensory systems. Such integration is essential for balance under conditions of altered somatosensory and/or visual input. Healthy individuals can integrate sensory information and select the most reliable cues for precise motor control, whereas concussed individuals may have difficulty maintaining equilibrium in challenging or discordant sensory environments when this process is impaired.

Despite evidence which suggests heading has no effect on postural stability $[4,17,23]$, it should be noted the conditions tested in prior studies may have failed to challenge the postural control system enough to elicit instability. Simply removing visual input has not been reported to cause instability in concussed populations [9] and the Clinical Test of Sensory Interaction and Balance is only validated for concussed populations who experience syncope [14]. A closely related test, the Sensory Organization Test (SOT), has been reported to be an effective diagnostic test for concussed populations by some [10] and ineffective by others [5]. Moreover, the SOT was not able to elicit postural instability in non-concussed football players after a high magnitude impact (e.g., >90 g) [21]. Thus, it is unlikely to be a sensitive test of postural instability following low force impacts (e.g., $20 \mathrm{~g}$ ) that occur as a result of heading.

The current findings may be due to the dynamic environmental conditions used, which were challenging enough to differentiate the healthy from the impaired postural system. It is already known that such dynamic discordant sensory input (e.g., an unstable support surface with a VE) can challenge even a healthy postural control system [29]. Our results indicate that discordant sensory input may be diagnostically sensitive following trauma induced by a bout of heading.

There is speculation about the mechanisms for dysfunction following trauma [4]. The rapid deceleration of the skull and brain during ball impact could replicate the type of physical trauma that affects underlying neural mechanisms in a concussion. However, given the low ball velocities used in this study it is unlikely the impact could cause large enough shearing forces to structurally damage neurons [3].

An alternative explanation for the postural deficits found in the current study could be result of a biochemical cascade [18] induced by the 10 subconcussive impacts which occurred over a relatively short period of time. According to a recently developed post-concussive biochemical cascade model, the timeline for post-concussive alterations in glucose metabolism shows that 


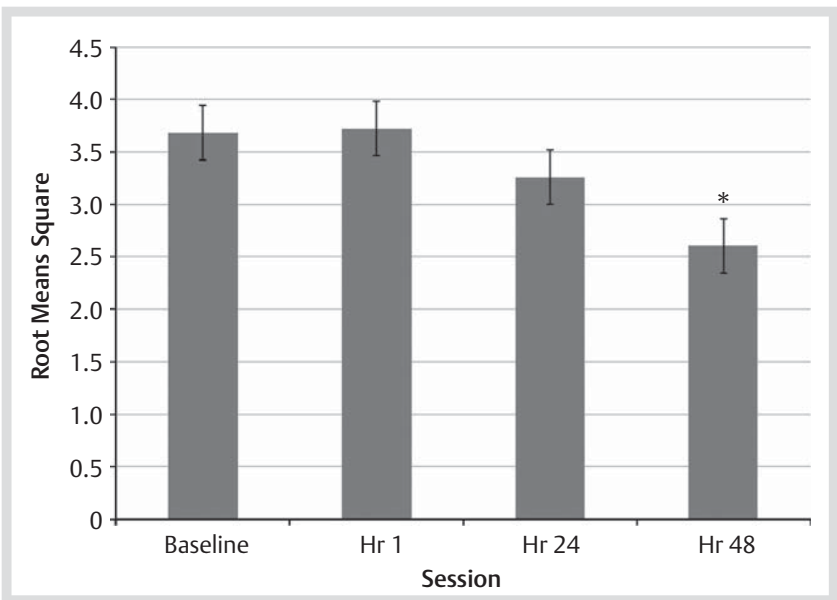

Fig. 5 AP COM means for time collapsed across group and condition. *Significant differences between time periods $(p<0.05)$.

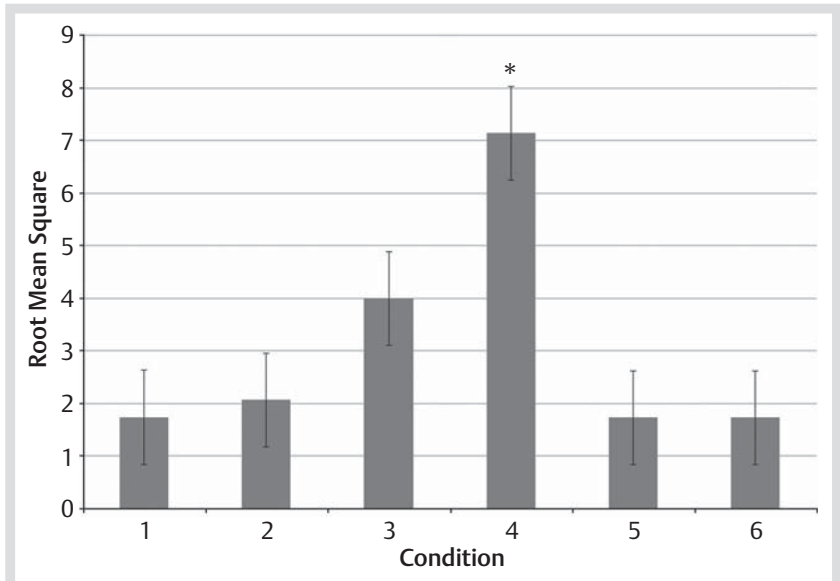

Fig. 6 AP COM means for condition collapsed across group and time. * Significant differences between conditions $(p<0.05)$.

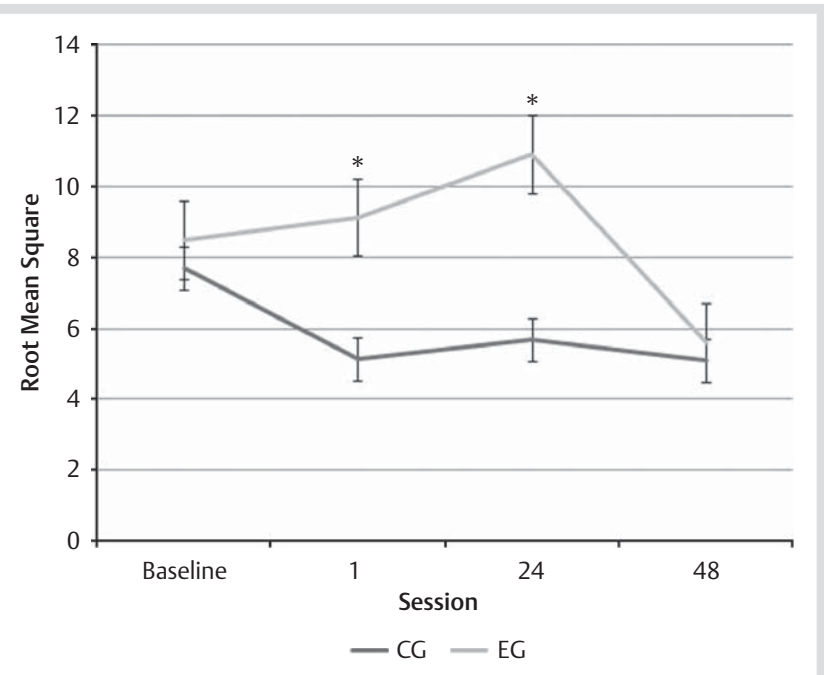

Fig. 7 AP COM group means for condition $4 .{ }^{*}$ Significant differences between groups $(p<0.05)$. within $30 \mathrm{~min}$ of injury increased localized cerebral glucose metabolism occurs, followed by hypometabolism after $6 \mathrm{~h}$, which can then last up to 5 days [2]. This glucose imbalance has been proposed previously as an explanation for post-concussive postural deficits [10].

Postural deficits found in this study are not as robust as postural deficits following a concussion, yet there is a similar, albeit truncated, recovery timeline with deficits appearing after the onset of glucose hypometabolism (i.e., hr 1), dissipating before $\mathrm{hr} 48$. This faster recovery period supports previous hypotheses that propose milder injuries (e.g., subconcussive trauma) may recover more rapidly from glucose hypometabolism than a concussion. Previous researchers may not have seen any postural deficits because post heading assessments were performed immediately after heading, thus, prior to glucose hypometabolism $[17,18]$.

Some limitations of our study are that we examined only the acute effects of heading and with a limited number of trials. Ball speed was not scaled according to gender which may play a factor due to different neck strengths; however, the effects may be minimal. Researchers have reported $10 \%$ higher head accelerations ( 20.2 vs. $18.2 \mathrm{~g}$ or $\sim 2 \mathrm{~g}$ difference) in females vs. male college-aged soccer players using ball speeds similar to ours $(22 \mathrm{mph})$. This difference was attributed to females exhibiting significantly less head mass and neck strength vs. their male counterparts [30]. Gender was controlled for in subject selection ( 2 females each in each group). The number of headers performed may have contributed to our results and future studies should perform 5 or 6 headers which is a more representative of game conditions. The protocol only addressed one aspect of neurological functioning. To be comprehensive future studies should also assess symptomatic, cognitive, and other motor domains using advanced methodologies such as biochemical markers and imaging techniques. Additionally, future studies should investigate the role of practice effects by training all subjects beforehand until a stable baseline is reached then randomly assigning subjects to the EG or CG.

This study did not address whether long-term soccer participation has a cumulative effect on neurological functioning. The effect of years of repetitive heading may lead to a cumulative degrading effect in some players that manifest similar to a concussion, a condition that would be not unlike dementia pugilistica. A header is difficult to perform, however if proper technique is taught early, the loading of the head may be controlled to prevent injury. Future longitudinal research examining cumulative effects is warranted to assure continued safe participation in soccer.

\section{Conclusions \\ $\nabla$}

Soccer participation is on the rise, especially in the US. With its high number of head injuries, evaluating the safety of the sport is paramount. This study is the first to report statistically significant alterations in postural control post heading. Even more symptoms may have been detected if the ball velocity $(11.2 \mathrm{~m} / \mathrm{s})$ was more representative of actual collegiate playing conditions $(\sim 30 \mathrm{~m} / \mathrm{s})$. Though our sample was small, EG and CG were similar in the most relevant characteristics, including familiarity with the postural stimuli, thus, it is unlikely the results are due to sampling error. The use of discordant sensory stimulation in an 
enriched large field-of-view VE may have increased our ability to detect postural differences between groups.

\section{Acknowledgements}

Supported by NIH-NIA grant AG26470 and NIH-NIDCD grant DC05235. We gratefully acknowledge VRCO, Inc., for the use of the CAVELIB to develop the VE.

\section{References}

1 Abreau F, Templer D, Schuyler B, Hutchison H. Neuropsychogical assessment of football players. Neuropsychology 1990; 4: 175-181

2 Barkoudarian G, Hovda H, Giza C. The molecular pathophysiology of concussive brain injury. Clin Sports Med 2011; 30: 33-48

3 Blumbergs $P$, Jones $N$, North J. Diffuse axonal injury in head trauma. J Neurol Neurosurg Psychiatry 1989; 52: 838-841

4 Broglio S, Guskiewicz K, Sell T, Lephart S. No acute changes in postural control after soccer heading. Br J Sports Med 2004; 38: 561-567

5 Cavanaugh J, Guskiewicz K, Giuliani C, Marshall S, Mercer V, Stergiou N. Detecting altered postural control after cerebral concussion in athletes with normal postural stability. Br J Sports Med 2005; 39: 805-811

6 Downs $D$, Abwender $D$. Neuropsychological impairment in soccer athletes. J Sports Med Phys Fitness 2002; 42: 103-107

7 Erlanger D, Kutner K, Barth J, Barnes R. Neuropsychology of sportsrelated head injury: Dementia Pugilistica to Post Concussion Syndrome. Clin Neuropsychol 1999; 13: 193-209

8 Guskiewicz K, Marshall S, Broglio S, Cantu R, Kirkendal D. No evidence of impaired neurocognitive performance in collegiate soccer players. Am J Sports Med 2002; 30: 157-162

9 Guskiewicz K, Perrin D, Gansneder B. Effect of mild head injury on postural stability in athletes. J Athl Train 1996; 31: 300-306

10 Guskiewicz K, Scott S, Marshall S. Postural stability and neuropsychological deficits after concussion in collegiate athletes. J Athl Train 2001; 36: 263-273

11 Haran F, Keshner E. Sensory reweighting as a method of balance training for labyrinthe loss. J Neurol Phys Ther 2008; 32: 186-191

12 Harriss DJ, Atkinson G. Update - Ethical standards in sport and exercise science research. Int J Sports Med 2011; 32: 819-821

13 Higgins M, Tierney RT, Caswell S, Driban J, Mansell J, Clegg S. Reliability of a functional heading impact testing model. J Sports Eng Tech 2009; 223: 117-123

14 Ingersoll C, Armstrong C. The effects of closed-head injury on postural sway. Med Sci Sport Exerc 1992; 24: 739-743

15 Jordan S, Green G, Galanty L. Acute and chronic brain injury in United States National Team soccer players. Am J Sports Med 1996; 24: 205-210

16 Kaminski T, Cousino E, Glutting J. Examining the relationship between purposeful heading in soccer and computerized neuropsychological test performance. Res Q Exerc Sport 2008; 79: 235-244
17 Matser J, Kessels A, Jordan B, Lezak M, Troost J. Neuropsychological impairment in amateur soccer players. JAMA 1999; 282: 971-973

18 Matser J, Kessels A, Jordan B, Lezak M, Troost J. Chronic traumatic brain injury in professional soccer players. Neurology 1998; 51: 791-796

19 Matser J, Kessels A, Lezak M, Troost J. A dose-response relation of headers and concussions with cognitive impairment in professional soccer players. J Clin Exp Neuropsychol 2001; 26: 770-774

20 Mangus B, Wallmann H, Ledford M. Analysis of postural stability in collegiate soccer players before and after an acute bout of heading multiple soccer balls. Sports Biomech 2004; 3: 209-220

21 McCaffrey M, Mihalik J, Crowell M, Shields E, Guskiewicz K. Measurement of head impacts in collegiate football players: clinical measures of concussion after high- and low-magnitude impacts. Neurosurg 2007; 61: 1236-1243

22 Putukian M, Echemendia R, Mackin S. The acute neuropsychological effects of heading in soccer: a pilot study. Clin Sports Med 2000; 10: $104-109$

23 Schmitt D, Hertel J, Evans T, Olsted L, Putukian M. Effect of an acute bout of soccer heading on postural control and self-reported concussion symptoms. Int J Sports Med 2004; 25: 326-331

24 Slaboda J, Barton J, Maitin I, Keshner E. Visual field dependence influences balance in patients with stroke. Conf Proc IEEE Eng Med Biol Soc 2009; $1147-1150$

25 Stalnake B, Tegner $Y$, Sojka P. Playing soccer increases serum concentrations of the biochemical markers of brain damage S-100B and neuron-specific enolase in elite players: a pilot study. Brain Inj 2004; 18: 899-909

26 Stalnake B, Ohlsson A, Tegner Y, Sojka P. Serum concentrations of two biochemical markers of brain tissue damage S-100B and neurone specific enolase are increased in elite female soccer players after a competitive game. Br J Sports Med 2006; 40: 313-316

27 Stephens R, Rutherford A, Potter D, Fernie G. Neuropsychological impairment as a consequence of football (soccer) play and football heading: a preliminary analysis and report on school students (13-16 years). Child Neuropsychol 2005; 11: 513-526

28 Straume-Naesheim T, Andersen T, Dvorak J, Bahr R. Reproducibility of computer based neuropsychological testing among Norwegian elite football players. Br J Sports Med 2005; 39 (S1): 64-69

29 Streepey J, Kenyon R, Keshner E. Visual motion combined with base of support width reveals variable field dependency in healthy young adults. Exp Br Res 2007; 176: 182-187

30 Tierney R, Higgins M, Caswell S, Brady J, McHardy K, Driban J, Darvish K. Sex differences in head acceleration during heading while wearing soccer headgear. J Ath Train 2008; 43: 578-584

31 Tysvaer A, Løchen E. Soccer injuries to the brain. A neuropsychologic study of former soccer players. Am J Sports Med 1991; 19: 56-60

32 Webbe F, Ochs $S$. Recency and frequency of soccer heading interact to decrease neurocognitive performance. Appl Neuropsychol 2003; 10: $31-41$

33 Witol A, Webbe F. Soccer heading frequency predicts neuropsychological deficits. Arch Clin Neuropsychol 2003; 18: 397-417 